\title{
Multi-institutional Positioning Test Feedback Dashboard for Aspiring Students
}

\author{
Katrien Verbert \\ KU Leuven \\ Department of Computer Science \\ Leuven, Belgium \\ [surname].[name]@kuleuven.be
}

Lessons Learnt from a Case Study in Flanders

\begin{abstract}
Our work focuses on a multi-institutional implementation and evaluation of a Learning Analytics Dashboards (LAD) at scale, providing feedback to $\mathrm{N}=337$ aspiring STEM (science, technology, engineering and mathematics) students participating in a region-wide positioning test before entering the study program. Study advisors were closely involved in the design and evaluation of the dashboard. The multi-institutional context of our case study requires careful consideration of external stakeholders and data ownership and portability issues, which gives shape to the technical design of the LAD. Our approach confirms students as active agents with data ownership, using an anonymous feedback code to access the LAD and to enable students to share their data with institutions at their discretion. Other distinguishing features of the LAD are the support for active content contribution by study advisors and $\mathrm{HT}_{\mathrm{E}} \mathrm{X}$ typesetting of question item feedback to enhance visual recognizability. We present our lessons learnt from a first iteration in production.
\end{abstract}

\section{CCS CONCEPTS}

- Information systems $\rightarrow$ Data analytics; • Human-centered computing $\rightarrow$ Information visualization; • Applied computing $\rightarrow$ Education;

\section{KEYWORDS}

learning analytics, student dashboard, positioning test, feedback, higher education, case study

\section{ACM Reference Format:}

Tom Broos, Katrien Verbert, Greet Langie, Carolien Van Soom, and Tinne De Laet. 2018. Multi-institutional Positioning Test Feedback Dashboard for Aspiring Students: Lessons Learnt from a Case Study in Flanders. In Proceedings of 8th International Learning Analytics and Knowledge Conference (LAK18). ACM, New York, NY, USA, 5 pages. https://doi.org/10.1145/ nnnnnnn.nnnnnnn

Permission to make digital or hard copies of all or part of this work for personal or classroom use is granted without fee provided that copies are not made or distributed for profit or commercial advantage and that copies bear this notice and the full citation on the first page. Copyrights for components of this work owned by others than ACM must be honored. Abstracting with credit is permitted. To copy otherwise, or republish, to post on servers or to redistribute to lists, requires prior specific permission and/or a fee. Request permissions from permissions@acm.org.

LAK18, March 2018, Sydney, Australia

(C) 2018 Association for Computing Machinery.

ACM ISBN 978-x-xxxx-xxxx-x/YY/MM...\$15.00

https://doi.org/10.1145/nnnnnnn.nnnnnnn

\author{
Greet Langie \\ Carolien Van Soom \\ Tinne De Laet \\ KU Leuven
}

Leuven Engineering and Science Education Centre

Leuven, Belgium

[surname].[name]@kuleuven.be

\section{INTRODUCTION}

Learning Analytics Dashboards (LADs) have been researched extensively over the past decade. LADs have been defined as "a single display that aggregates multiple visualizations of different indicators about learner(s), learning process(es), and/or learning context(s)" [15]. LADs often rely on a variety of data sources, including resource use, social interactions, and time spent [20], to support different stakeholders with insight into learning processes. A recent review that compares work of the LAK and the OLM (Open Learner Model) communities argues that assessment data is often not included in typical LADs: only $37 \%$ of the surveyed LADs included some form of assessment data, while it is at the core of every OLM system that was reviewed. Assessment data has shown significant effects on learning and decision making [1]. Although not a prerequisite for impactful dashboards, inclusion of assessment data has several benefits: it is widely available and privacy concerns are limited [3].

In contrast to many existing studies that are often conducted at a small scale [2], our work targets LA at scale with university- and nation-wide deployments. A first public iteration of our dashboard was used by $\mathrm{N}=337$ students in transition from secondary to higher education after the September '17 edition of the Flemish regionwide position test for a STEM (science, technology, engineering, and mathematics) study program.

The context of our work is challenging and involves not only our own institution, but also external stakeholders from other (competing) universities. Our target audience of aspiring STEM students has no formal relationship with the institution yet at the time of distribution of the LAD. Nevertheless our approach allows them to share their data electronically with an institution of choice at their own discretion after enrollment. We consider the active involvement of study advisors (SAs) in the design and evaluation process a strong point and facilitate co-creation as an important LAS feature.

The contribution of our work is twofold: we describe a number of simple but effective technical solutions in response to a complex multi-institutional stakeholder setting with related data ownership issues. Second, we show lessons learnt from the deployment at scale in three large universities that used our LAD to provide feedback to aspiring students. To evaluate the LAD, we make use of the Evaluation Framework for Learning Analytics (EFLA), recently validated as an instrument to assess and compare LA tools [14]. 


\section{CONTEXT}

Providing feedback to students in transition. The dashboard presented in this study aims at providing feedback to students in the transition from secondary to higher education (HE) in STEM programs. Both from an academic and social perspective, this transition is challenging [17]. Students have to adapt their study strategy to the new context of HE, without a priori knowing how and what they have to adapt. Lacking an objective means of comparison, social comparison theory [5] suggests that students in transition search for comparison to peers.

Earlier research has shown that prior achievements of students in secondary school are the best predictors for student performance in HE STEM programs $[11,13]$. Many studies confirm that both higher school leaving matriculation test scores (e.g., Scholastic Aptitude Test - SAT; American College Testing - ACT; or A-level score) and higher Grade Point Average (GPA) contribute to higher GPA and better persistence in a first-year STEM program. Moreover, soft skills such as learning and studying skills are positively related to study success [12].

Feedback has been a proven powerful tool for improving student achievement, but its effectiveness depends on the type of feedback and the circumstances under which feedback is given [6]. Feedback during the transition from secondary to higher education is considered pivotal regarding student motivation, confidence, retention, and success $[8,9]$.

Positioning test. Unlike most other countries, the transition to $\mathrm{HE}$ in Flanders (the Dutch-speaking region of Belgium) does not involve any formal selection methods or entrance criteria ${ }^{1}$ : (1) no national school-leaving examinations (i.e., matriculation exams) are organized at the end of secondary education, and (2) no entrance examinations are organized by HE institutions. As a consequence, there is a large degree of heterogeneity of incoming students in terms of prior knowledge, attitudes, and skills. This results in an overall bachelor drop-out rate of around $40 \%$ in STEM programs at KU Leuven. Since 2011, the Flemish universities offering engineering bachelor programs have joined efforts for organizing a 'positioning test', a diagnostic test for the candidate students' ability to solve math problems $[18,19]$. The multiple choice test is organized in the summer between the end of secondary education and the start of HE. Since 2015, a facultative questionnaire including three scales (concentration, motivation, and time management) from the Learning and Studying skills Inventory (LASSI) [21] is appended to the positioning test. Candidate students are not subscribed to a university program yet, which provides particular challenges for the feedback and students' data privacy. Feedback should be provided as soon as possible, considering it should still influence the students study choice, and using an online format, as there is no other way to contact the students. Moreover, upon request students can book an appointment with a SA of any of the participating universities. To this end, the student's data and test results should be made available to the SA.

\footnotetext{
${ }^{1}$ Except for study programs in Medicine, Dentistry, and Arts Education - see [10] for detailed information.
}

Stakeholders. Rolling out iterations of a LA intervention at scale requires careful consideration of the different stakeholders involved. We identified three key groups.

(1) Aspiring students are both the data subjects and main target group of the feedback dashboard. Given that participation to the positioning test is voluntary and that the result is not binding, a fair assumption would be that many of the aspiring students have some interest in receiving detailed feedback. At the time of test participation, a formal student-institution relationship is not yet established. Aspiring students may choose to enroll at any of the organizing universities. This makes the target group difficult to involve in the initial design phase of the intervention.

(2) Study advisors (SAs) are involved in the test organization and provide face-to-face feedback to students. They are in a position close to aspiring and first-year students, combined with the experience to link positioning test scores and student profiles to study results in the upcoming year. Our dashboard influences their work, as it replaces the existing one-size-fits-all feedback document and interferes with the in-person feedback sessions to which a number of students subscribe to discuss test results.

(3) Management of all involved universities are shaping the conditions for LA interventions. While a fully crossinstitutional design process may not be feasible due to time and budget constraints, we considered it to be important to keep the respective management groups well-informed, to provide an open line of communication and to assure the neutrality of the dashboard and underlying data analysis.

\section{DASHBOARD SYSTEM DESIGN}

In response to the pre-enrollment status of the dashboard's data subject and target group, the close involvement of SAs and the rollout across several universities, several features were added to the dashboard. These are discussed in this section, which also explains the visual and textual content of the LAD.

Feedback code pseudonymization. Many authors have voiced their concerns about data privacy in LA. Slade and Prensloo [16] provide a integrated overview from a socio-critical perspective. They propose a set of grounding principles as a starting point for a context-aware application. One of these principles concerns viewing students as active agents for learning analytics, as opposed to merely passive data producers or intervention targets. Within the context of providing feedback to future students - these are students that at the time of data collection did not yet engage in a formal relationship with the participating institutions - questions about data ownership are even more pronounced than in many other case studies.

Our case study implements the conceptual framework for deidentification for LA described by Khalil \& Ebner [7]. Test and survey results are collected and processed by a shared organization trusted by the participating universities. Before delivery to the LA case study, these data are stripped from personal identifiers and characteristics that allow for straightforward re-identification. A pseudonymization process replaces the identifiers with a surrogate key of the format $10 \mathrm{ir}-9 \mathrm{c} 7 \mathrm{~s}-41 \mathrm{jn}-18$ (dummy example), the 'feedback code'. In addition to a prefix and optional spaces or 


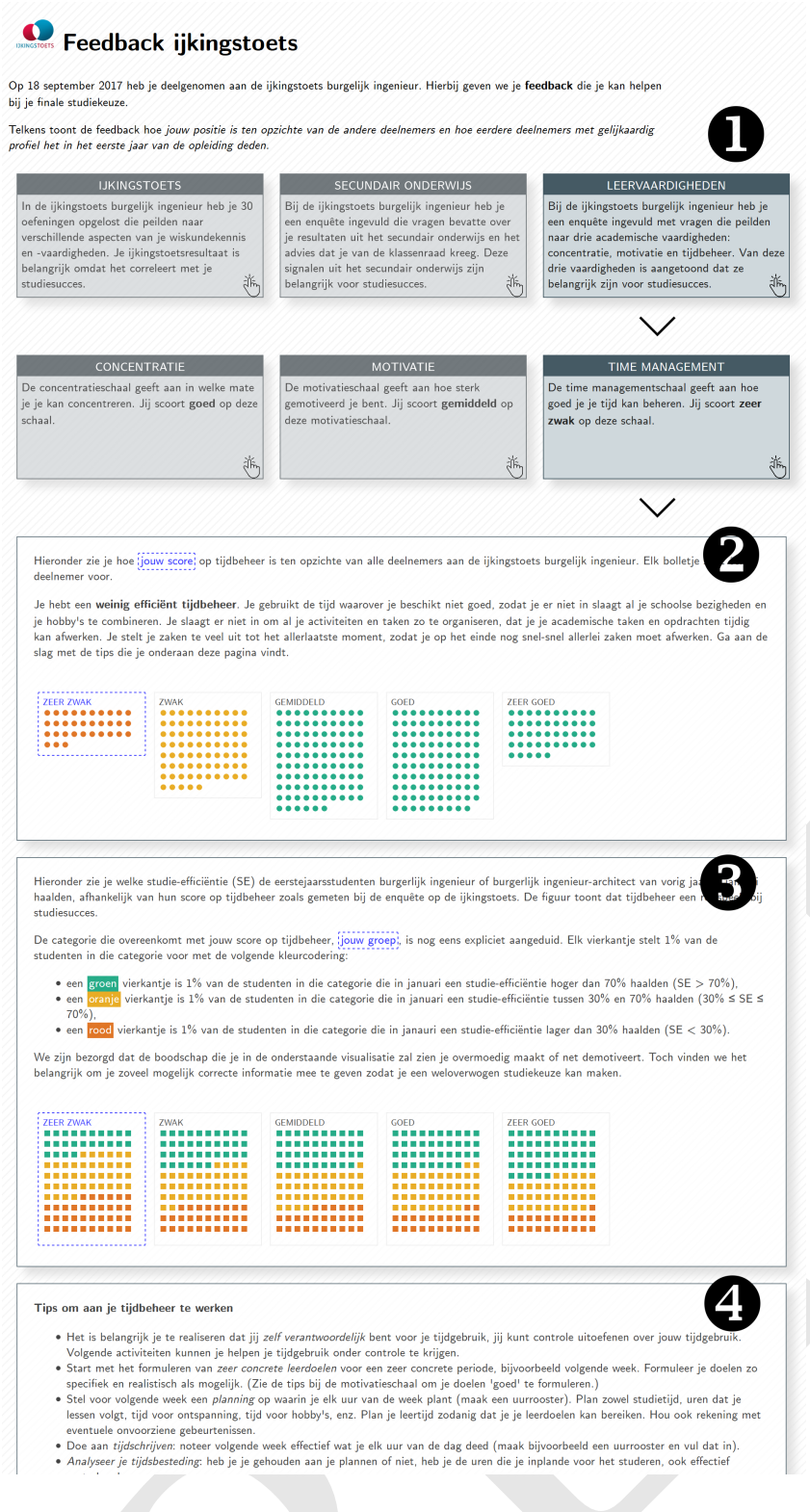

Figure 1: Full-screen view of the dashboard for a user navigation to the main category 'learning skills' (1), drilling down further to the feedback about 'time management'. First, the individual student's score is put into context (2) and compared to the scores of other students participating in the test. Second, the score is compared (3) to last years' students and their rate of study success in the program. At the bottom (4) a series of tips is provided for the student wishing to improve, alongside links to resources available in the different universities.

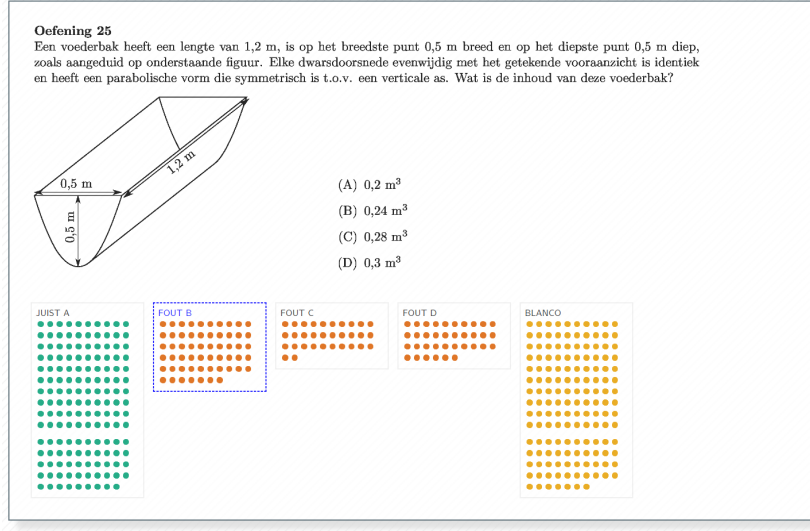

Figure 2: Extract of the dashboard view, focusing on the feedback about a question in the position test. The question and its associated figures and formulas are displayed using identical typesetting as on the (paper) test document to improve visual recognition. The dot chart shows the number of test participants selecting answers A-D or blank respectively. The correct answer is displayed using green dots, wrong and blank answers using red and orange dots respectively. The blue border around answer $B$ indicates that this was the dashboard user's (incorrect) answer.

dashes as chunk delimiters, the key format contains ten random alphanumeric digits (a-z; 0-9), resulting in $36^{10}$ possible combinations.

Students who participated in the positioning test, receive an institutional-neutral invitation email with their general result for the positioning test (passed / failed and score). The message includes a personal feedback code and a link to the dashboard, which is openly accessible via HTTPS. An opening page prompts the anonymous visitor to enter a feedback code. The data access API of the dashboard system is throttled to a limited number of simultaneous connections and imposes an artificial delay of 1 second before replying to requests, to obstruct brute-force guessing of feedback codes to obtain (anonymous) test results. Students who prefer to discuss the results with a SA of one of the involved universities, have the option to share their feedback code. This approach confirms the active agent position and data ownership of the student. In principle, SAs could (maliciously) keep track of individual codes shared with them, but systematic access to student data is prevented.

Data layer. The data layer of the dashboard is separated from the presentation layer discussed below. Data is served in JSON format in response to a POST HTTPS request containing a valid feedback key. Outside of the intended dashboard's scope, this approach provides each of the participating institutions (or any other party) a possibility to request the feedback data for a given student through an automated process, given that this student chooses to share the individual feedback code with the institution. This possibility demonstrates future portability of the feedback data and how students could be put in control of such portability in a very lightweight manner. Each of the universities could implement their 
own LA instruments consuming this data that predates the enrollment of the student. While our approach could be further enhanced by the use of standards, leading e-learning standardization efforts seem to be focused mainly on learning activity. The Experience API (xAPI), for example "lacks specific support for any student profile information" [4]

Content contribution by study advisors. The feedback dashboard was designed in close collaboration with SAs involved in the positioning tests. Once the dashboard was given shape, a preview version was made available to them. Using a set of dummy feedback codes, SAs could run different scenarios, seeing the dashboard through the eyes of students with profiles of different strength. SAs were able to adapt each of the dashboard's components ('cards') in response to specific scenario's by simply CTRL+double-clicking the part to be edited. This opens a scenario-based parametrization interface. All information is saved and edited in Markdown format, extended by a set of dashboard-specific keywords, e.g. anumberCorrect@ for the number of positioning test questions answered correctly by a student or @mathGroup@ for the secondary school math result group the student belongs to. Some keywords, like @green@ and @red@ are available to include an in-line legend for the charts within the text.

Presentation layer. To structure the information in the LAD, a hierarchical representation using 'cards' is used (see Figure 1). Users start the navigation at the top, selecting one of the feedback categories they want to explore further. The dashboard offers three categories of feedback to the student: (1) feedback directly related to the positioning test $\rightarrow$ test score summary and item-level feedback; (2) feedback related to the secondary school $\rightarrow$ class council's advice and Math scores; (3) feedback about meta-cognitive abilities of the student $\rightarrow$ concentration, motivation, and time management.

Question typesetting. The test copies handed out to students during the formal test moments, are composed using the $\mathbf{L T}_{\mathbf{E}} \mathbf{X}$ typesetting system, which makes it possible to embed mathematical equations, charts, and figures in a way that is familiar to faculty staff. This results in a typical, clean typesetting on paper. To enhance the visual recognizability of the test questions in the feedback dashboard, they are shown in precisely the same way on screen as on the original test copy, as illustrated for one question on Figure 2. A procedure extracts the $\mathrm{LTE}_{\mathrm{E} X}$ source code of each test question and compiles them to images that are embedded in the dashboard.

\section{EVALUATION}

As a test run, students who took the July ' 17 edition of the positioning test, did not receive a direct link to the dashboard yet. Instead, a limited group of students that made an appointment with a SA were invited to bring their personal feedback code to the meeting. Upon entry of this code, the feedback dashboard became available as an instrument to facilitate the conversation between students and SAs. For a first qualitative study of dashboard usage, some of these conversations were recorded $(\mathrm{N}=12)$ and coded. Following a positive evaluation of the dashboard test run in the safe environment of the student-advisor interaction, it was approved for general availability in a student-only context for mid-September test participants. Together with the test results, a link to the dashboard was

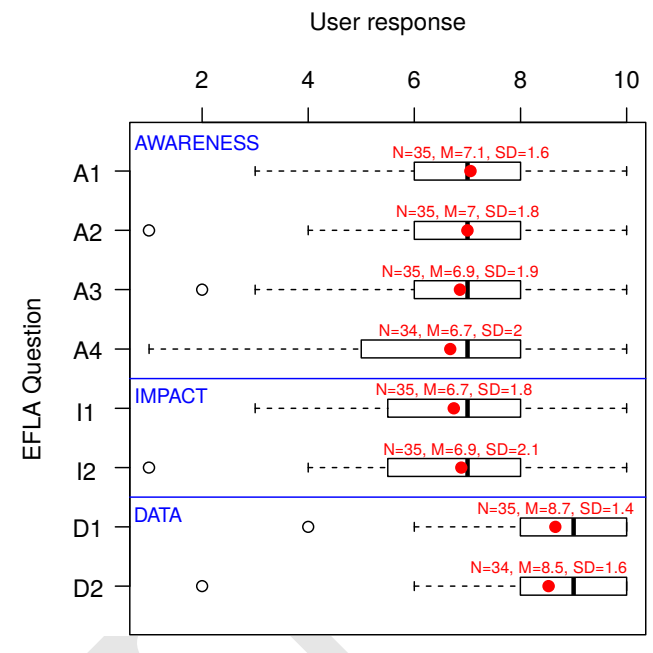

Figure 3: User responses on a scale of 1-10 on the eight EFLA evaluation questions, further grouped into three categories Awareness \& Reflection, Impact, and Data.

included in the email message to 421 students, of which $80 \%$ clicked through to the $\mathrm{LAD}(\mathrm{N}=337)$. Once the dashboard was offered to aspiring students, additional user evaluations were conducted and usage behavior was tracked.

To evaluate student's perception of the LAD, students were asked to complete an on-screen questionnaire implementing the Evaluation Framework for Learning Analytics (EFLA, version 4), a validated instrument [14] to assess and compare LA tools. The questions were translated to Dutch and adapted minimally to reflect the topic of the dashboard. In consultation with SAs, the data related questions D1 and D2 were placed at the bottom of the questionnaire, resulting in the following list of items: A1 This dashboard makes me more aware of my current study situation. A2 (...) makes me forecast my possible study situation, with/without change in my learning behavior. A3 (...) stimulates me to think about my past learning behavior. A4 (...) stimulates me to change my learning behavior or study trajectory, and/or strengthens me in my current learning behavior or study trajectory. I1 (...) stimulates me to study more efficiently (as in: making sure to work in the right way). I2 (...) stimulates me to study more effectively (as in: making sure to reach the target, in any way). D1 It is clear to me which data are being collected to assemble this dashboard. D2 It is clear to me why the data shown in this dashboard are being collected.

The dashboard has an overall EFLA score ${ }^{2}$ of 72/100 (see Figure 3 for item scores), which we consider to be acceptable for a first iteration available at scale. Questions A1-A4 and I1-I2 are grouped into the Awareness \& Reflection and Impact dimensions respectively, with scores $66 / 100$ and $65 / 100$, illustrating that the dashboard performs without major flaws, but with room for improvement. The data dimension outperforms with a score of 84/100.

Complementing the qualitative study of dashboard usage within student-advisor sessions and the quantitative feedback using EFLA,

\footnotetext{
${ }^{2}$ See http://www.laceproject.eu/evaluation-framework-for-la/ for concise instructions to calculate overall and dimensional EFLA scores.
} 


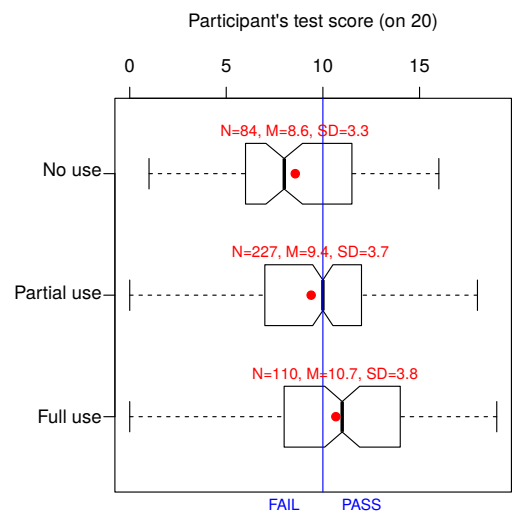

Figure 4: Participant's test score vs. dashboard use

usage tracking was used as a third means to build understanding of how aspiring students are using the LAD. Several events like clicking and scrolling behavior and total usage time were monitored. A particular finding (see Figure 4) is the difference in mean and median positioning test scores of aspiring students who (1) did not use the dashboard at all; (2) did access the dashboard, but did not view all feedback categories; (3) viewed the entire dashboard. This may indicate that students with a stronger profile also tend to be more open to holistic feedback, while students with a weaker profile may be more focused on the feedback specifically linked to the positioning test outcome.

\section{DISCUSSION AND FUTURE WORK}

This paper presented the approach and lessons learnt from a case study using a LAD to provide feedback to aspiring students after participation in an official positioning test organized in cooperation by three Flemish universities.

In response to some of the specific stakeholder requirements originating from the multi-institutional context and the absence of a formal relationship between target group and institutions, we presented some simple, but in our experience effective features of the LAD. Special effort was taken to enable involvement of SAs.

The work presented here is still in preliminary phase. A more thorough analysis of the usage traces may help to identify additional usage patterns and different behavior in function of students' profiles. Forthcoming findings should be discussed in focus groups with SAs and (meanwhile) first-year students to gather insights to improve a future iteration of the dashboard (July '18 edition). Current EFLA scores provide a baseline to formulate and test improvement targets.

While it would be interesting to see if the LAD has actual behavioural impact on students, ethical concerns may restrict a quantitative approaches to verification.

An aspect that is underexposed in this paper is the difference in usage pattern when the LAD is used to facilitate the student-advisor conversation and when the same LAD is used - as intended - by students on their own. It would be interesting to explore if and how these different modes contribute differently to the Awareness \& Reflection and Impact dimensions of the EFLA.

\section{ACKNOWLEDGMENTS}

This research is co-funded by the Erasmus+ program of the European Union (562167-EPP-1-2015-1-BE-EPPKA3-PI-FORWARD).

\section{REFERENCES}

[1] Robert Bodily, Judy Kay, Vincent Aleven, Dan Davis, Ioana Jivet, Franceska Xhakaj, and Katrien Verbert. 2018. Open learner models and learning analytics dashboards: a systematic review. In Proceedings of the Eight International Conference on Learning Analytics \& Knowledge. ACM.

[2] Robert Bodily and Katrien Verbert. 2017. Review of research on student-facing learning analytics dashboards and educational recommender systems. IEEE Transactions on Learning Technologies (2017), 1-14.

[3] Sven Charleer, Andrew Vande Moere, Joris Klerkx, Katrien Verbert, and Tinne De Laet. 2017. Learning Analytics Dashboards to Support Adviser-Student Dialogue. IEEE Transactions on Learning Technologies (2017), 1-12.

[4] Ángel Del Blanco, Ángel Serrano, Manuel Freire, Iván Martínez-Ortiz, and Baltasar Fernández-Manjón. 2013. E-Learning standards and learning analytics. Can data collection be improved by using standard data models?. In Global Engineering Education Conference (EDUCON), 2013 IEEE. IEEE, 1255-1261.

[5] Leon Festinger. 1954. A theory of social comparison processes. Human relations 7, 2 (1954), 117-140.

[6] John Hattie and Helen Timperley. 2007. The power of feedback. Review of educational research 77, 1 (2007), 81-112.

[7] Mohammad Khalil and Martin Ebner. 2016. De-identification in learning analytics. Journal of Learning Analytics 3, 1 (2016), 129-138. https://doi.org/10.18608/jla. 2016.31.8

[8] O. Lisa, M. Morag, P. Margaret, H. Nuala, M. Geraldine, R. Seamus, and M. Conor. 2016. Technology-Enabled Feedback in the First Year: A synthesis of the literature. Technical Report. http://www.teachingandlearning.ie/wp-content/uploads/2016/ 10/SynthesisoftheLiterature2016.pdf

[9] David Nicol. 2009. Assessment for learner self-regulation: enhancing achievement in the first year using learning technologies. Assessment \& Evaluation in Higher Education 34, 3 (2009), 335-352.

[10] Maarten Pinxten, Bieke De Fraine, Wim Van Den Noortgate, Jan Van Damme, Tinneke Boonen, and Gudrun Vanlaar. 2015. 'I choose so I am': a logistic analysis of major selection in university and successful completion of the first year. Studies in Higher Education 40, 10 (2015), 1919-1946.

[11] Maarten Pinxten, Tinne De Laet, Carolien Van Soom, and Greet Langie. 2015. Fighting increasing drop-out rates in the STEM field: The European readySTEMgo Project. In Proceedings of the 43rd Annual SEFI Conference. 1-8.

[12] Maarten Pinxten, Carolien Van Soom, Christine Peeters, Tinne De Laet, Pal Pacher, Peter Hockicko, and Greet Langie. 2016. Learning and study strategies of incoming science and engineering students. A comparative study between three institutions in Belgium, Slovakia, and Hungary. In Proceedings of the 44rd Annual SEFI Conference. $1-8$.

[13] Michelle Richardson, Charles Abraham, and Rod Bond. 2012. Psychological correlates of university students' academic performance: a systematic review and meta-analysis. Psychological bulletin 138, 2 (2012), 353.

[14] Maren Scheffel, Hendrik Drachsler, Christian Toisoul, Stefaan Ternier, and Marcus Specht. 2017. The proof of the pudding: examining validity and reliability of the evaluation framework for learning analytics. In European Conference on Technology Enhanced Learning. Springer, 194-208.

[15] Beat A Schwendimann, María Jesús Rodríguez-Triana, Andrii Vozniuk, Luis P Prieto, Mina Shirvani Boroujeni, Adrian Holzer, Denis Gillet, and Pierre Dillenbourg. 2016. Understanding learning at a glance: An overview of learning dashboard studies. In Proceedings of the Sixth International Conference on Learning Analytics \& Knowledge. ACM, 532-533.

[16] Sharon Slade and Paul Prinsloo. 2013. Learning Analytics. American Behavioral Scientist 57, 10 (2013), 1510-1529. https://doi.org/10.1177/0002764213479366

[17] V. Tinto. 2012. Leaving College: Rethinking the Causes and Cures of Student Attrition. University of Chicago Press.

[18] Jef Vanderoost, Carolien Van Soom, Greet Langie, Johan Van den Bossche, Riet Callens, Joos Vandewalle, and Tinne De Laet. 2015. Engineering and science positioning tests in Flanders: powerful predictors for study success?. In Proceedings of the 43rd Annual SEFI Conference. 1-8.

[19] Joos Vandewalle and R Callens. 2013. A positioning test mathematics in Flanders for potential academic engineering students. In Proc. of the 41st SEFI Conference. $1-8$.

[20] Katrien Verbert, Sten Govaerts, Erik Duval, Jose Luis Santos, Frans Van Assche, Gonzalo Parra, and Joris Klerkx. 2014. Learning dashboards: an overview and future research opportunities. Personal and Ubiquitous Computing 18, 6 (2014), 1499-1514.

[21] Claire E Weinstein, SA Zimmerman, and DR Palmer. 1988. Assessing learning strategies: The design and development of the LASSI. Learning and study strategies: Issues in assessment, instruction, and evaluation (1988), 25-40. 